\title{
Is body fat percentage a better measure of undernutrition in newborns than birth weight percentiles?
}

\author{
Angela E. Carberry 1,2, Camille H. Raynes-Greenow ${ }^{2}$, Robin M. Turner ${ }^{2}$, Lisa M. Askie ${ }^{3}$ and Heather E. Jeffery ${ }^{1,2}$
}

BACKGROUND: Undernutrition in neonates increases the risk of serious morbidities. The objective of this study was to describe neonatal morbidity associated with low body fat percentage (BF\%) and measure the number of undernourished neonates defined by BF\% and compare this with birth weight percentiles (<10th).

METHODS: Eligibility included term ( $\geq 37 \mathrm{wk}$ ) neonates. BF\% measurements were undertaken by air displacement plethysmography. Data on neonatal outcomes were extracted from medical records and used to develop a measure of neonatal morbidity. We assessed the association between neonatal morbidity and population-based birth weight percentiles compared with the BF\% measurements.

RESULTS: Five hundred and eighty-one neonates were included. Low BF\% was defined by 1 SD below the mean and identified in 73 per 1,000 live births. Neonatal morbidity was found in 3.4\% of neonates. Birth weight percentile was associated with neonatal morbidity (odds ratio (OR): 1.03 (95\% confidence interval (Cl): 1.01, 1.05); $P=<0.001)$. BF\% was associated with a higher risk of neonatal morbidity (OR: 1.30 (95\% Cl: 1.15, 1.47); $P=<0.001$ ).

CONCLUSION: In this population, measuring BF\% is more closely associated with identification of neonates at risk of neonatal morbidity as compared with birth weight percentiles. $\mathrm{BF} \%$ measurements could assist with identifying neonates who are appropriately grown yet undernourished and exclude small neonates not at risk.

A ccurately determining the nutritional status of newborns is a major public health problem $(1,2)$. The World Health Organization estimates that undernutrition contributes to more than half of all child deaths in the developing countries by leaving the child more vulnerable to severe consequences from common infections (3) and severe neurological complications due to insufficient energy (substrates) in the perinatal period (4). Worldwide, the majority of neonatal deaths occur on the first day and within the first week after birth. Furthermore, undernourished neonates who survive are at risk of long-term health outcomes, including hypertension, stroke, type 2 diabetes, obesity, and cardiovascular disease (5-8). Neonatal undernutrition, or "wasting", is a clinical diagnosis often characterized by diminished subcutaneous tissues and underlying muscles with loose wrinkled skin of the arms, legs, elbows, and knees $(1,8)$.

Size at birth and specifically birth weight is commonly used to identify neonates at risk from fetal undernutrition. Small-term neonates and preterm neonates have difficulty in maintaining normal blood glucose concentration and are at risk of hypoglycemia and its consequences as glucose is the major energy substrate for neonates (9). During pregnancy, the fetus is entirely dependent on placental transfer of maternal glucose. Following birth, neonates' transition to oral intake through breastfeeding or breast milk substitutes provides glucose (10). However, before oral feeding is established, mobilization of body fat is very important for the neonate as body fat provides alternative substrates for the newborn's glucose-dependant brain and other tissues. Measuring body fat percentage (BF\%) in neonates may be an accurate measure of risk of insufficient energy and, or hypoglycemia. A variety of methods are used to detect neonatal BF\% including total body electrical conductivity, bioelectrical impedance, and dual-energy X-ray absorptiometry (11). However, these techniques are generally impractical for wide use in a neonatal population because they are relatively invasive, time consuming, and may involve some radiation exposure $(11,12)$. Recently, a new technology using air displacement plethysmography (ADP) has become available to noninvasively, accurately, and quickly measure $\mathrm{BF} \%$ in infants from birth to 6 mo of age. ADP has been validated against the four-compartment model and biological and physical phantoms (13) and is considered the criterion method for determining BF\% in neonates (14-18). Several studies have investigated the $\mathrm{BF} \%$ as the indicator of neonatal nutritional status using ADP (13,19-21). However, the neonatal morbidity associated with $\mathrm{BF} \%$ at birth has not yet been described.

Therefore the aim of this study was to (1) describe neonatal morbidity associated with low BF\% (2), measure the number of undernourished neonates defined by low BF\%, and (3) examine the association between neonatal morbidity in population-based birth weight percentiles compared with neonatal BF\% measurement. 


\section{RESULTS}

There were 815 term neonates born during the study period. Of the 782 eligible neonates, 581 were enrolled in the study with $75 \%$ recruitment (Figure 1). Neonatal, maternal, and paternal demographic characteristics are presented in Tables 1 and 2.

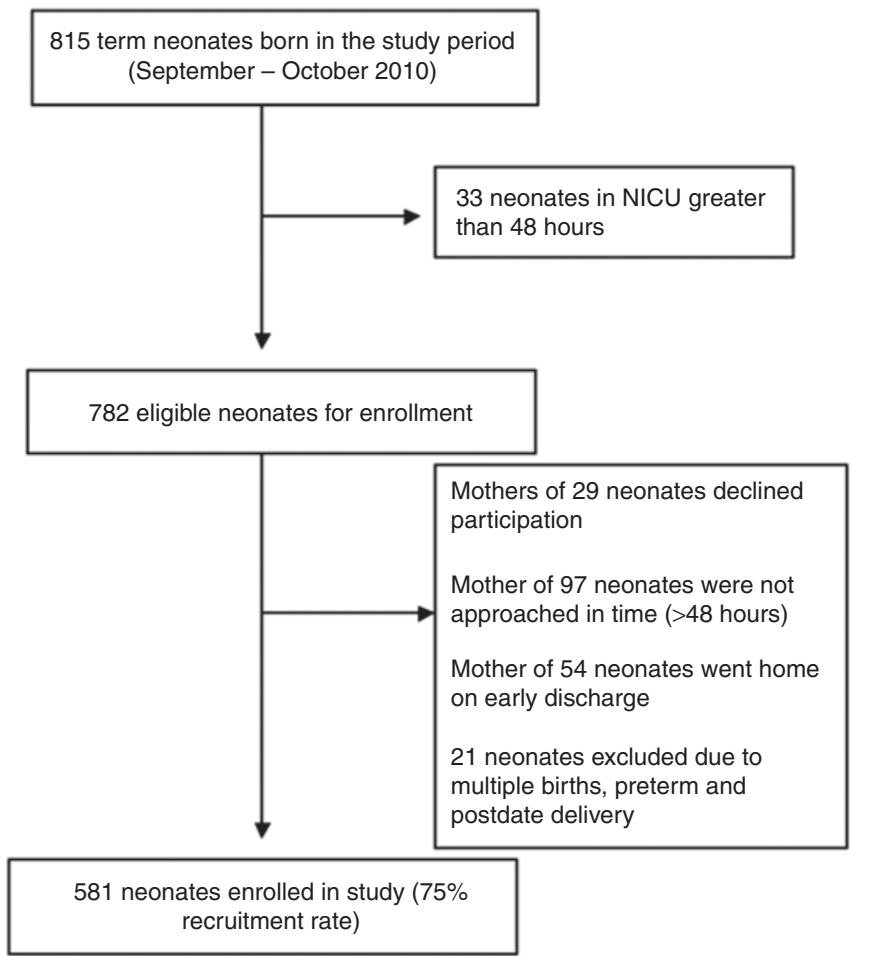

Figure 1. Recruitment flow chart.

Table 1. Neonatal characteristics of the 581 neonates enrolled in the study

\begin{tabular}{lc}
\hline & Neonatal characteristics $(n=581)$ \\
\hline Gestational age (wk; mean (SD)) & $39.5(1.2)$ \\
Birth weight (kg; mean (SD)) & $3,435(465)$ \\
Length (cm; mean (SD)) & $49.7(2.0)$ \\
Sex (male) & $51.8 \%$ \\
Parity & \\
$\quad$ Primipara (\%) & $56.5 \%$ \\
Multipara (\%) & $43.5 \%$ \\
Ethnicity & \\
Caucasian & $63.3 \%$ \\
Asian & $33.1 \%$ \\
Other & $3.6 \%$ \\
BF\% at birth (mean (SD)) & $9.22(4.33)$ \\
\% Breastfed at birth & $96.7 \%$ \\
SGA $(n)$ & 45 \\
LGA $(n)$ & 61 \\
\hline
\end{tabular}

$\mathrm{BF} \%$, body fat\%; LGA, large for gestational age; SGA, small for gestational age.

\section{Development of a Composite Neonatal Morbidity}

The results of the univariate logistic regression identified hypothermia, poor feeding, and extended length of stay were mostly associated with being small for gestational age (SGA) vs. appropriate for gestational age. These three markers were combined to create the measure of undernutrition, and thus neonates were defined as having morbidity if they had all three of these markers. There were $20(3.4 \%)$ neonates found to have neonatal morbidity based on these conservative criteria.

\section{Birth Weight Percentile as a Predictor of Neonatal Morbidity}

Percentile birth weight was associated with the neonatal morbidity with an odds ratio (OR) of 1.03 (95\% confidence interval $(\mathrm{CI}): 1.01,1.05 ; P=0.0019)$ indicating that for every one percentile decrease in weight the OR of neonatal morbidity increased. Model discrimination measured by the area under the receiver operating characteristic (ROC) curve was 0.71 , indicating moderate discrimination.

\section{BF\% and Fat Mass as a Predictor of Neonatal Morbidity}

Undernourished neonates classified by low BF\% $(<1$ SD below the mean) had a higher risk of neonatal morbidity OR of 1.30 (95\% CI: $1.15,1.47 ; P \leq 0.001)$ demonstrating that for every

Table 2. Maternal and paternal characteristics of the 581 neonates enrolled in the study

\begin{tabular}{lcc}
\hline & $\begin{array}{c}\text { Maternal } \\
\text { characteristics } \\
(n=581)\end{array}$ & $\begin{array}{c}\text { Paternal } \\
\text { characteristics } \\
(n=581)\end{array}$ \\
\hline Age (years; mean (SD)) & $32(5.3)$ & $35(6.1)$ \\
Highest education level & & \\
Year 10 or less & $3.6 \%$ & $4.0 \%$ \\
High school & $24.8 \%$ & $26.1 \%$ \\
Tertiary & $68.8 \%$ & $64.1 \%$ \\
Missing & $2.8 \%$ & $5.8 \%$ \\
BMI (kg/m²; mean (SD)) & $23.0(4.6)$ & \\
Smoking (\%) & $3.8 \%$ & \\
Hypertensive disease (\%) & & \\
Gestational (\%) & $1.3 \%$ & \\
Preeclampsia (\%) & $5.0 \%$ & \\
Eclampsia (\%) & $1.2 \%$ & \\
Diabetes & $0.2 \%$ & \\
Gestational (\%) & $11.0 \%$ & \\
Type Il (\%) & $0.7 \%$ & \\
Type I (\%) & $0.3 \%$ & \\
Type of delivery & & \\
NVD (\%) & $13.1 \%$ & \\
C/S elective (\%) & & \\
C/S emergency (\%) & & \\
Instrumental delivery & & \\
\hline C/S, & & \\
\hline & & \\
\hline
\end{tabular}

$C / S$, cesarean section; NVD, normal vaginal delivery. 
$1 \%$ decrease in $\mathrm{BF} \%$ the odds of morbidity increased. The area under the ROC curve was 0.79 showing moderate discrimination of neonatal morbidity. When we used fat mass (FM), the association with neonatal morbidity was very similar with an area under the ROC curve of 0.78 .

The logistic regression model showed that $\mathrm{BF} \%$ was significantly different to birth weight percentiles $(P=0.0084)$ providing evidence that BF\% (OR: 1.25 (95\% CI: 1.08, 1.45)) is associated with poor neonatal outcomes than birth weight percentiles (OR: 1.01 (95\% CI: 1.01, 1.03)). However, as the ROC comparison is much less sensitive to differences (i.e., requires very large ORs to show a significant improvement in ROC (22)), the comparison of BF\% and birth weight percentiles using ROCs was not significant $(P=0.17)$. Figure 2 shows the improvement in the discrimination of neonatal morbidity using BF\% and FM compared with percentile weight (SGA). Due to the extremely high correlation between FM and BF\% (as is expected) we cannot test for differences in a statistical model, in our sample BF\% has the higher OR and ROC though the differences are minor. There was high correlation between neonatal BF\% and population-based percentile weight $(r=0.60 ; P<$ 0.001 ), indicating that the two variables were measuring similar but not identical aspects of neonatal anthropometry.

Maternal BMI was not associated with poor neonatal outcomes $(P=0.37)$ nor with predicting BF\% $(P=0.35)$. BMI did not confound the relationship between $\mathrm{BF} \%$ and neonatal morbidity (change in OR for BF\%: 1.2\%). When maternal height and weight were included in a model predicting neonatal morbidity, maternal weight was weakly associated $(P=0.09)$ and maternal height was not associated $(P=0.86)$ with neonatal morbidity. Of note, mothers $<50 \mathrm{~kg}$ were more likely to have neonates with neonatal morbidity (OR: 4.3 (95\% CI: 1.4, 13.8); $P=0.014)$ compared with mothers with weight between 50 and

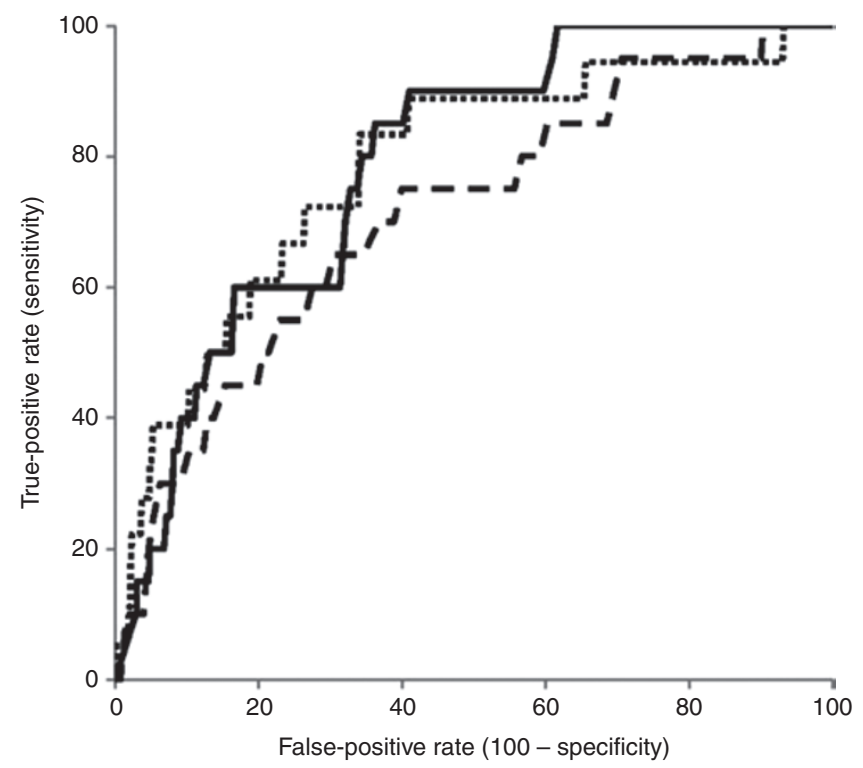

Figure 2. The receiver operating characteristic (ROC) curves for neonatal morbidity predicted separately from each of body fat (BF\%), fat mass (FM), and birth weight percentiles. Solid black line, BF\%; smaller dashed line, FM; and larger dashed lined, birth weight percentiles.
$64.9 \mathrm{~kg}$. Height was however, associated with predicting $\mathrm{BF} \%$ $(P=0.005)$, specifically those women who were less than $160 \mathrm{~cm}$ in height had a lower average $\mathrm{BF} \%$, compared with women who were between 160 and $174.9 \mathrm{~cm}$ tall, after adjustment for maternal weight. When maternal height and weight were included in the model for $\mathrm{BF} \%$ predicting neonatal morbidity, the association between $\mathrm{BF} \%$ and neonatal morbidity was not confounded by these variables (the OR for BF\% changed by only $2.1 \%$ ).

\section{Thresholds for Targeting High-Risk Neonates}

Using the standard screening threshold of $<10$ th percentile (SGA), sensitivity was 30\% (95\% CI: $12,54 \%)$ and specificity was 93\% (95\% CI: 91, 95\%) for detection of neonatal morbidity. Using the standard screening threshold for BF\% at $1 \mathrm{SD}$ cutoff the sensitivity was $45 \%$ (95\% CI: $23,68 \%)$ and the specificity was $85 \%$ (95\% CI: $82,88 \%$ ). For 1.5 SD below the mean for males and females, the sensitivity was $26 \%$ and the specificity was $93 \%$. At 2 SD below the mean, the sensitivity was $16 \%$ and the specificity was $96 \%$. Ideally, to be useful in determining neonatal morbidity the $\mathrm{BF} \%$ threshold should have maximum sensitivity (i.e., not miss any undernourished neonates) but not at the expense of specificity (to minimize unnecessary testing of neonates). The $1 \mathrm{SD}$ below the mean threshold, in comparison with 1.5 and $2 \mathrm{SD}$, provided the best balance of low $\mathrm{BF} \%$ cut off between maximum sensitivity without unnecessarily low specificity.

\section{Incidence of Appropriately Grown Neonates With Low BF\%}

The distribution of the neonatal $\mathrm{BF} \%$ and the incidence of neonates who had low BF\% $(<1 \mathrm{SD}$ below mean) measured by $\mathrm{ADP}$ was 73 per 1,000 births (Figure 3 ). This equates to 333 undernourished neonates born per year in our setting. The number of neonates with low BF\% identified by using the $<10$ th birth weight percentile cutoff was 29 (32\%) neonates; thus $68 \%$ were clinically unrecognized or appropriately grown

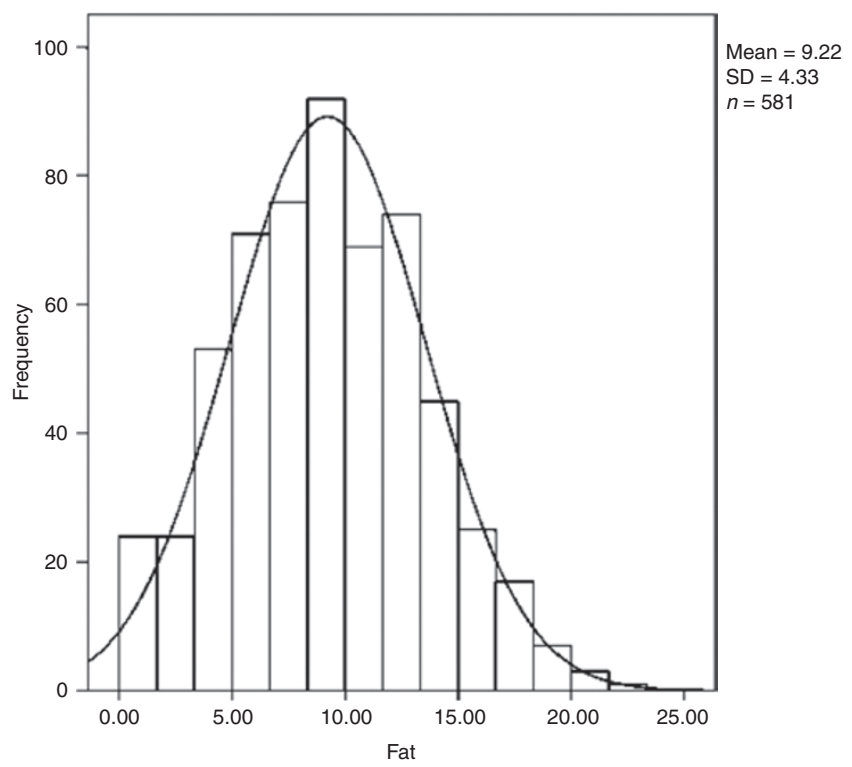

Figure 3. The distribution of $\mathrm{BF} \%$ in 581 neonates. $\mathrm{BF} \%$, body fat $\%$. 
yet undernourished. Of the 45 neonates $<10$ th percentile, 16 had normal BF\% (36\%).

\section{DISCUSSION}

To our knowledge, this is the first study that has used ADP to measure BF\% at birth, identify a threshold for neonatal BF\% and investigate the association with neonatal morbidity compared with birth weight percentiles. We found that those neonates with low BF\% (1 SD below the mean) had a higher risk of neonatal morbidity based on a surrogate measure of morbidity which included hypothermia, extended length of stay, and poor breastfeeding in comparison with the traditional approach of using population-based growth charts using logistic regression, but was not found to be significantly better at identifying neonates at risk using the appropriate ROC analysis. That is BF\% in our sample was better at discriminating neonatal morbidity compared with the usual method of birth weight percentiles, and specifically $<10$ th (SGA), but we had insufficient evidence to show that it was significantly better. These findings are directly relevant to clinicians globally as the use of BF\% measurements could assist with identifying neonates at risk of neonatal morbidity associated with undernutrition. We found that the incidence of low BF\% was 73 per 1,000 births in apparently healthy term neonates.

These results suggest that the risk of associated neonatal morbidity was slightly higher in the low BF\% neonates compared with the standard method $(<10$ th percentile) for detecting risk of hypoglycemia. The neonatal morbidity measure for undernutrition used in our study was based on previous research (23-29) and clinical input of the pathophysiology of growth restricted neonates. In particular, Kramer et al. (23) found that growth restriction was associated with numerous adverse metabolic and neonatal outcomes. In addition, Walther and Ramaekers (30) found that asphyxia, hypoglycemia, and hypothermia occurred significantly more frequently in wasted neonates with a low Ponderal Index $\left(\right.$ weight $/$ height $\left.^{3} \times 100\right)$ and the combination of wasting and underweight pointed to a higher incidence of neonatal morbidity. Our findings show low rates of neonatal morbidity in our population (3.4\%); we believe this is due to three reasons. First, we excluded any neonate that was in the neonatal intensive care unit for greater than $48 \mathrm{~h}$ as access to the neonate was impractical thereby excluding the neonates at most risk. Second, we had very high rates of breastfeeding initiation and support. The recent accreditation of our setting as a Baby Friendly Hospital Initiative (31) before the study commencing maximized high compliance with breastfeeding guidelines. As breast milk is a ready source of alternative ketogenic substrates this may have reduced the number of neonates at risk of the variables used to identify neonatal morbidity. Last, the number of neonates with neonatal morbidity was defined by an 'and' rule in which a neonate had to have all three outcomes which may have under estimated the true rate of neonatal morbidity. This final point is, however, the strength of our study and increases our confidence in our results.

Currently available tools for detection of neonatal undernutrition include the clinical assessment of nutritional status score (CANSCORE) which consists of nine physical detectable signs of undernutrition and anthropometric measures, such as the Ponderal Index. The CANSCORE has been investigated by Adebami and Owa (1) who found that by using the CANSCORE as the objective measure in 442 terms, singleton neonates, $18.8 \%$ were undernourished. The authors compared different indictors of undernutrition and found that using an intrauterine growth standard alone, $49.4 \%$ of the neonates with neonatal undernutrition would have been missed while Ponderal Index would also have missed $61.4 \%$ of neonates (1). Other research has investigated perinatal outcomes using the CANSCORE to identify undernourished preterm neonates, and found that early neonatal morbidities including hypoglycemia, feeding intolerance and infection were higher in undernourished neonates compared with those appropriately grown (32). The CANSCORE is a purely clinical assessment requiring determination of physical characteristics of the newborn, thus heavily dependent on clinician or health worker skills for assessment (8). Neither the clinical assessment nor Ponderal Index methods have used a highly accurate tool to assess nutritional status such as ADP to determine sensitivity and specificity. An important aspect of the $\mathrm{ADP}$ is that it is an objective measurement that provides accurate clinical measurements without observer error. These findings are important as previously there has been no accurate, validated and objective measurement for detection and monitoring of risks associated with substrate depletion.

The strengths of our study include that it was a population representative sample with a good recruitment rate $(75 \%)$, and would be widely generalizable to other similar high-income settings where ADP equipment is available. ADP has been validated against many reference models including the fourcompartment model (14) and thus provides noninvasive measurement to determine body composition including BF\% in neonates. In addition, the fluctuating hydration status of the neonate during the first week of life is accounted for during the BF\% measurements. The advantage of measuring body composition is that neonatal $\mathrm{BF} \%$ can provide an estimate of risk of undernutrition and likely neonatal morbidity such as failure of alternative substrates for brain metabolism, hypothermia, and feeding difficulties. Weaknesses of our study include that we were not able to use neonatal blood glucose levels, which best indicate hypoglycemia and thus undernutrition, as these are not performed in all neonates as part of routine practice. However, we chose an objective, surrogate composite measure of neonatal morbidity associated with undernutrition, which included variables that are supported by the pathophysiological evidence to identify neonates who would have been at risk of undernutrition. Our composite neonatal morbidity measure was conservative as neonates had to have all three of the outcomes to be defined as morbid, and those neonates with a single morbid variable were not included in outcomes. Furthermore, our sample size does not enable us to develop models based on these individual outcomes. Although the variables used in our neonatal morbidity outcome are associated with neonatal morbidity due to undernutrition they may also indicate other neonatal conditions. Maternal height and weight were self reported which may increase the risk of bias, 
although for most mothers these were cross-checked with their antenatal data, which was measured for the majority of women in the first trimester of pregnancy. ADP technology is relatively new and although the equipment approximates the cost of an ultrasound machine which is commonly available, it is not yet available in all clinical settings. Finally, some parents initially found the use of the machine daunting.

In conclusion, $\mathrm{BF} \%$ measurements in newborns were shown to be more closely associated with undernutrition using logistic regression. However, the very low rates of neonatal morbidity in this study are suggestive of but do not provide definitive results as to whether BF\% is a better method of identifying neonates at risk of undernutrition compared with the population-based birth weight percentile of SGA ( $<10$ th) using ROC curve comparisons. These findings are clinically relevant and potentially could be applied as an accurate screening program in settings where ADP is available. In particular, this study establishes that $\mathrm{BF} \%$ may be a better method of detecting the nutritional status of appropriate for gestational age neonates who are undernourished and at risk yet unrecognized and thus not screened for risk of hypoglycemia. Identifying neonates with low BF\% could improve opportunities for early intervention for neonates at risk of morbidity and mortality associated with undernutrition and exclude those not at risk who are $<10$ th percentile but constitutionally small.

\section{METHODS}

\section{Subjects}

Using a cross-sectional design subjects were recruited from term, singleton, neonates born at Royal Prince Alfred Hospital (RPAH) between September and October 2010. RPAH is a major public teaching hospital in Sydney, Australia, with a predominantly inner urban, multicultural population and $>5,000$ births per year. Eligible subjects were well, term neonates (37-42 wk gestation) within the first $48 \mathrm{~h}$ of birth. Exclusions included neonates with major congenital abnormalities, multiple births, preterm neonates, or admissions to the neonatal intensive care unit for greater than $48 \mathrm{~h}$ as illness or invasive monitoring prevented measurement. The greatest loss in BF\% occurs on day 3 and therefore to standardize this measurement we included only neonates measured within $48 \mathrm{~h}$ of birth (33). The sample size was based on the main study question of estimating the number of neonates with low $\mathrm{BF} \%$ with a minimum sample size of 384 required to provide a $95 \% \mathrm{CI} \pm 3 \%$. This was based on the assumption of $10 \%$ prevalence of neonates with low BF\% giving 95\% CI: 7, 13\%.

\section{Measurements}

Neonatal data included weight (kilograms (kg), length, sex, ethnicity, and gestational age (weeks). SGA was defined as $<10$ th percentile birth weight and appropriate for gestational age was defined as between the 10 and 90th percentile birth weight, large for gestational age as $>90$ th percentile birth weight (34).

$\mathrm{BF} \%$ measurements were performed using ADP (PEA POD; COSMED, Concord, CA). Before testing, the neonates' clothes were removed and a head cap was applied or hair smoothed to reduce the amount of air behaving isothermically. Neonatal weight was measured using the integrated scale to the nearest gram. Mass and volume calibrations took into account the presence of two hospital identification bracelets and an umbilical cord clip on each neonate at birth. The BF\% was computed by software integral to the PEA POD system (version 3.1.3) based on a two compartment model of fat and fatfree compartments. Within the volume calculation, corrections were applied for air within the lungs and in close proximity to the subject's skin, which behaves isothermically. Neonatal BF\% is calculated from body density assuming the density of fat to be $0.9007 \mathrm{~kg} / \mathrm{l}$. Age- and gender-specific densities of fat-free mass were computed based on the data of Fomon et al. (35). For neonates up to 6-d-old, this fat-free mass density calculation allows for the greater hydration of fat-free mass in the first week of life (21). FM and fat-free mass were computed from weight and BF\% (13).

Two trained personnel measured neonatal length to $\sim 0.1 \mathrm{~cm}$ heel to crown using an Easy-Glide Bearing Infantometer (Perspective Enterprises, Portage, MI). The intraclass correlation coefficient was 0.968 showing good inter-rater reliability (36). Birth weight was measured using the digital scales on the PEA POD to within $0.1 \mathrm{~g}$. The method for measuring length and anthropometry was standardized by skills-based educational methods by a neonatologist $(\mathrm{HJ})$ and competency was confirmed before data collection (37).

During the neonatal measurement, mothers completed a short interviewer-administered questionnaire to collect data on preexisting medical conditions, maternal and paternal demographic details. Maternal BMI (weight $(\mathrm{kg}) /$ height $(\mathrm{m})^{2}$ ), prepregnancy weight and height were collected as potential confounders. Categories of height $(\mathrm{cm})$ and weight $(\mathrm{kg})$ were developed where the thresholds for low weight and height were close to the lower quartile of the data including $<50 \mathrm{~kg}$ and $<160 \mathrm{~cm}$. Medical records were assessed in all neonates with the exception of two whose data were unavailable.

\section{Data Analysis}

Development of a composite neonatal morbidity. Blood glucose is a key indicator of neonatal hypoglycemia due to substrate deficiency and thus can indicate undernutrition. However, blood glucose testing is not routinely performed on all neonates in the neonatal period, and hypoglycemia is relatively rare in developed countries, therefore this data was not available. In addition, undernourished neonates are more likely to have poor perinatal outcomes such as stillbirth, neonatal death, low Apgar scores (24), operative delivery or cesarean section for reasons of fetal distress (25), hypoglycemia, hypothermia (26), respiratory distress syndrome, intraventricular hemorrhage, necrotizing enterocolitis, increased length of stay (27), and poor breastfeeding $(28,29)$. Many of these outcomes are also relatively rare in our setting, and data on these outcomes are insufficient for cross-sectional studies. Therefore, based on the prevalence of these variables and discussions with clinicians and the literature (23-29) we developed a surrogate composite measure of neonatal morbidity associated with undernutrition. This included fetal distress, Apgar scores, hypothermia, length of stay, and poor feeding (Appendix 1). We used univariate logistic regression to develop this surrogate morbidity measure. As the current standard method for screening neonates at risk of neonatal morbidity and mortality is based on their birth weight percentiles, we chose $<10$ th percentile (SGA) as the morbidity outcome to identify these factors. Our univariate regression included the five outcomes to identify which variables were associated with SGA compared with appropriate for gestational age neonates. We used an "and" rule, that is an neonate was defined as having neonatal morbidity if he or she had all of the outcomes significantly related to SGA status.

Birth weight percentile as a predictor of neonatal morbidity. Univariate logistic regression was used to assess whether birth weight percentiles (a continuous variable) were associated with neonatal morbidity. The sensitivity and specificity at varying thresholds of weight percentiles were used to calculate a ROC curve. The ability of birth weight percentiles to discriminate neonatal morbidity was measured using the area under the ROC curve. The difference in association between $\mathrm{BF} \%$ and birth weight percentiles was investigated in two ways: by testing for a difference between these two variables in a logistic regression model; and by comparing the ROC curves (38).

BF\% and FM as a predictor of neonatal morbidity. To determine if $\mathrm{BF} \%$ is a more appropriate indicator of neonatal morbidity we conducted a univariate logistic regression assessing the association between BF\% (a continuous variable) and neonatal morbidity and estimated the area under the ROC curve. Similarly, we assessed FM (a continuous variable) and neonatal morbidity using a logistic regression model to estimate the area under the ROC curve. We also 
investigated whether maternal BMI, height, or weight confounded any association between $\mathrm{BF} \%$ and neonatal morbidity.

Thresholds for targeting high-risk neonates. In clinical practice birth weight thresholds are used for identifying neonates at high risk of morbidity. We investigated how well the commonly used threshold of $<10$ th birth weight percentile (SGA) compared with BF\% identified neonatal morbidity using sensitivity and specificity. There is no standard threshold for $\mathrm{BF} \%$ so we investigated three thresholds for BF\% for identifying neonatal morbidity again calculating sensitivity and specificity at each threshold. These thresholds were 1 SD below the mean (4.2\% for males and 5.8\% for females), 1.5 SDs below the mean $(2.1$ and $3.6 \%$, respectively) and 2 SDs below the mean $(<1$ and $1.5 \%$, respectively). In this context, it is important to find the highest sensitivity threshold (thus identifying neonatal morbidity) but not at the expense of reduced specificity that would increase the number of healthy neonates receiving unnecessary blood glucose testing.

All analyses were conducted in SPSS version 19 software (SPSS, Chicago, IL), SAS 9.2 software (SAS, Cary, NC), and STATA 11 (STATA, College Station, TX).

The study was approved by the Human Research Ethics Committees of Royal Prince Alfred Hospital and the University of Sydney (HREC/09/RPAH645, SSA/09/RPAH646, and University of Sydney Ref. No. 12732). Informed parental written consent was obtained, and participation was voluntary.

\section{APPENDIX 1}

\section{Outcomes Used for the Composite Neonatal Morbidity}

1. Fetal distress leading to operative delivery and/or thick meconium at birth,

2. Low Apgar scores ( $<7$ at $5 \mathrm{~min}$ ) leading to resuscitation at birth,

3. Hypothermia $\left(<36.5^{\circ} \mathrm{C}\right.$ or $\left.97.7^{\circ} \mathrm{F}\right)$ at any time,

4. Longer length of hospital stay than standard clinical practice ( $>3 \mathrm{~d}$ for normal vaginal delivery and instrumental delivery and $>5 \mathrm{~d}$ for cesarean section), and

5. Poor feeding based on three criteria with at least two of the following: (i) frequency ( $<3$ first $24 \mathrm{~h},<6$ next $24 \mathrm{~h}$ ); (ii) poor breastfeeding (or bottle feeding) codes (<code $4 \times 3$ ) (codes ranged from 1 to 6 , where 1 was defined by "breast offered but does not attach" and 6 was defined as "long feed with good nutritive sucking"); (iii) expressed breast milk or formula (greater than twice) if breastfed.

\section{ACKNOWLEDGMENTS}

The PEA POD donors included TENIX (Sydney, Australia) and an anonymous donor to the University of Sydney. The authors thank Royal Prince Alfred Hospital medical and nursing staff and Lucia Wang, Cheryl Au, Elizabeth Hayles, and Erin Donnelley for assisting with data collection. We thank the parents for their assistance in this project.

\section{STATEMENT OF FINANCIAL SUPPORT}

R.M.T. was supported by National Health and Medical Research Council (NHMRC, Sydney, Australia) program grant 633003 to the Screening \& Test Evaluation Program. C.H.R.-G. was supported by an NHMRC Early Career Fellowship grant 511481. L.M.A. is supported by an NHMRC Career Development Fellowship grant 571121. A.E.C. is supported by an Australian Postgraduate PhD Award.

Disclosure: There were no conflicts of interest to declare when completing this study.

\section{REFERENCES}

1. Adebami OJ, Owa JA. Comparison between CANSCORE and other anthropometric indicators in fetal malnutrition. Indian J Pediatr 2008;75:439-42.

2. Lawn JE, Cousens S, Zupan J; Lancet Neonatal Survival Steering Team. 4 million neonatal deaths: when? Where? Why? Lancet 2005;365:891-900.
3. Bryce J, Boschi-Pinto C, Shibuya K, Black RE; WHO Child Health Epidemiology Reference Group. WHO estimates of the causes of death in children. Lancet 2005;365:1147-52.

4. Grantham-McGregor SM, Walker SP, Chang S. Nutritional deficiencies and later behavioural development. Proc Nutr Soc 2000;59:47-54.

5. Barker DJ, Eriksson JG, Forsén T, Osmond C. Fetal origins of adult disease: strength of effects and biological basis. Int J Epidemiol 2002;31:1235-9.

6. Wells JC, Chomtho S, Fewtrell MS. Programming of body composition by early growth and nutrition. Proc Nutr Soc 2007;66:423-34.

7. Salihoglu O, Karatekin G, Ilhan R, Nuhoglu A. Placental findings in malnourished term neonates. Pediatr Int 2009;51:494-7.

8. Sankhyan N, Sharma VK, Singh S. Detection of fetal malnutrition using “CAN score". Indian J Pediatr 2009;76:903-6.

9. Gustafsson J. Neonatal energy substrate production. Indian J Med Res 2009;130:618-23.

10. Cowett RM, Loughead JL. Neonatal glucose metabolism: differential diagnoses, evaluation, and treatment of hypoglycemia. Neonatal Netw 2002;21:9-19.

11. Rigo J. Body composition during the first year of life. Nestle Nutr Workshop Ser Pediatr Program 2006;58:65-76; discussion 76-8.

12. Deierlein AL, Thornton J, Hull H, Paley C, Gallagher D. An anthropometric model to estimate neonatal fat mass using air displacement plethysmography. Nutr Metab (Lond) 2012;9:21.

13. Carberry AE, Colditz PB, Lingwood BE. Body composition from birth to 4.5 months in infants born to non-obese women. Pediatr Res 2010;68:84-8.

14. Ellis KJ, Yao M, Shypailo RJ, Urlando A, Wong WW, Heird WC. Bodycomposition assessment in infancy: air-displacement plethysmography compared with a reference 4-compartment model. Am J Clin Nutr 2007;85:90-5.

15. Ma G, Yao M, Liu Y, et al. Validation of a new pediatric air-displacement plethysmograph for assessing body composition in infants. Am J Clin Nutr 2004;79:653-60.

16. Sainz RD, Urlando A. Evaluation of a new pediatric air-displacement plethysmograph for body-composition assessment by means of chemical analysis of bovine tissue phantoms. Am J Clin Nutr 2003;77:364-70.

17. Urlando A, Dempster P, Aitkens S. A new air displacement plethysmograph for the measurement of body composition in infants. Pediatr Res 2003;53:486-92.

18. Yao M, Nommsen-Rivers L, Dewey K, Urlando A. Preliminary evaluation of a new pediatric air displacement plethysmograph for body composition assessment in infants. Acta Diabetol 2003;40 Suppl 1:S55-8.

19. Andersen GS, Girma T, Wells JC, Kæstel P, Michaelsen KF, Friis H. Fat and fat-free mass at birth: air displacement plethysmography measurements on 350 Ethiopian newborns. Pediatr Res 2011;70:501-6.

20. Fields DA, Gilchrist JM, Catalano PM, Giannì ML, Roggero PM, Mosca F. Longitudinal body composition data in exclusively breast-fed infants: a multicenter study. Obesity (Silver Spring) 2011;19:1887-91.

21. Lingwood BE, Storm van Leeuwen AM, Carberry AE, et al. Prediction of fat-free mass and percentage of body fat in neonates using bioelectrical impedance analysis and anthropometric measures: validation against the PEA POD. Br J Nutr 2012;107:1545-52.

22. Grund B, Sabin C. Analysis of biomarker data: logs, odds ratios, and receiver operating characteristic curves. Curr Opin HIV AIDS 2010;5:4739.

23. Kramer MS, Olivier M, McLean FH, Willis DM, Usher RH. Impact of intrauterine growth retardation and body proportionality on fetal and neonatal outcome. Pediatrics 1990;86:707-13.

24. Clausson B, Gardosi J, Francis A, Cnattingius S. Perinatal outcome in SGA births defined by customised versus population-based birthweight standards. BJOG 2001;108:830-4.

25. de Jong CL, Gardosi J, Dekker GA, Colenbrander GJ, van Geijn HP. Application of a customised birthweight standard in the assessment of perinatal outcome in a high risk population. Br J Obstet Gynaecol 1998;105:531-5.

26. Pallotto EK, Kilbride HW. Perinatal outcome and later implications of intrauterine growth restriction. Clin Obstet Gynecol 2006;49:257-69. 


\section{Articles $\mid$ Carberryet al.}

27. Gilbert WM, Danielsen B. Pregnancy outcomes associated with intrauterine growth restriction. Am J Obstet Gynecol 2003;188:1596-9; discussion 1599-601.

28. Rogers IS, Emmett PM, Golding J. The incidence and duration of breast feeding. Early Hum Dev 1997;49 Suppl:S45-74.

29. Scott JA, Landers MC, Hughes RM, Binns CW. Factors associated with breastfeeding at discharge and duration of breastfeeding. J Paediatr Child Health 2001;37:254-61.

30. Walther FJ, Ramaekers LH. The ponderal index as a measure of the nutritional status at birth and its relation to some aspects of neonatal morbidity. J Perinat Med 1982;10:42-7.

31. Naylor AJ. Baby-Friendly Hospital Initiative. Protecting, promoting, and supporting breastfeeding in the twenty-first century. Pediatr Clin North Am 2001;48:475-83.

32. Korkmaz A, Teksam O, Yurdakök M, Yigit S, Tekinalp G. Fetal malnutrition and its impacts on neonatal outcome in preterm infants. Turk J Pediatr 2011;53:261-8.
33. Roggero P, Giannì ML, Orsi A, et al. Neonatal period: body composition changes in breast-fed full-term newborns. Neonatology 2010;97:13943.

34. Beeby PJ, Bhutap T, Taylor LK. New South Wales population-based birthweight percentile charts. J Paediatr Child Health 1996;32:512-8.

35. Fomon SJ, Haschke F, Ziegler EE, Nelson SE. Body composition of reference children from birth to age 10 years. Am J Clin Nutr 1982;35(5 Suppl):1169-75.

36. Wood AJ, Raynes-Greenow CH, Carberry AE, Jeffery HE. Neonatal length inaccuracies in clinical practice and related percentile discrepancies detected by a simple length-board. J Paediatr Child Health 2013;49:199203.

37. Jeffery HE, Henderson-Smart DJ, Hill DA. Competency-based learning in neonatology. Med Educ 1996;30:440-4.

38. DeLong ER, DeLong DM, Clarke-Pearson DL. Comparing the areas under two or more correlated receiver operating characteristic curves: a nonparametric approach. Biometrics 1988;44:837-45. 